\title{
Crystal structure of the YTH domain of YTHDF2 reveals mechanism for recognition of N6-methyladenosine
}

Cell Research (2014) 24:1493-1496. doi:10.1038/cr.2014.152; published online 21 November 2014

\section{Dear Editor,}

N6-methyladenosine $\left(\mathrm{m}^{6} \mathrm{~A}\right)$ has been demonstrated to be ubiquitous in several types of eukaryotic RNAs, including messenger RNA (mRNA), transfer RNA (tRNA), ribosomal RNA (rRNA), long non-coding RNA (lncRNA), and small nuclear RNA (snRNA) [1]. The recent discoveries of RNA $\mathrm{m}^{6} \mathrm{~A}$ methyltransferase complex METTL3/METTL14/WTAP and demethylases FTO and ALKBH5 prove the reversibility of m6A modification [2-6]. This modification plays important roles in various biological processes, including circadian rhythms [7], RNA splicing [8], yeast meiosis [9], and embryonic stem cell self-renewal [10]. Two recent studies show that YTH domain family 2 (YTHDF2) and other YTHDF proteins preferentially bind to $\mathrm{m}^{6} \mathrm{~A}$-containing $\mathrm{mRNA}$ in vivo and in vitro and regulate localization and stability of the bound mRNA $[8,11]$. YTHDF2 is also known to be involved in development of acute myeloid leukemia [12]. YTHDC1 (splicing factor YT521-B), another YTH domain-containing protein, is known to play an important role in Emery-Dreifuss muscular dystrophy. While the function of YTHDF2 in the regulation of mRNA stability has been explored, the molecular mechanism for specific recognition of $\mathrm{m}^{6} \mathrm{~A}$ by the $\mathrm{YTH}$ domain remains elusive.

YTHDF 2 consists of a $\mathrm{C}$-terminal YTH domain (designated as $\mathrm{YTH}^{\mathrm{YTHDF} 2}$ ), which specifically binds to $\mathrm{m}^{6} \mathrm{~A}$-containing RNA ( $\mathrm{m}^{6} \mathrm{~A}-\mathrm{RNA}$ ) with a preference for those containing a consensus motif of $\mathrm{G}\left(\mathrm{m}^{6} \mathrm{~A}\right) \mathrm{C}[11]$. The YTH domains are highly conserved in YTH domain-containing proteins, including YTHDF1-3, YTHDC1, YTHDC2 (CsA-associated helicase-like protein), Mmil (Schizosaccharomyces pombe), and MRB1 (Saccharomyces cerevisiae), suggesting an important function of the YTH domain across species (Figure 1A).

To verify the specific recognition of $\mathrm{m}^{6} \mathrm{~A}-\mathrm{RNA}$ by $\mathrm{YTH}^{\mathrm{YTHDF} 2}$, we performed fluorescence polarization (FP) assays and electrophoretic mobility shift assays (EMSA) using purified $\mathrm{YTH}^{\mathrm{YTHDF} 2}$, unmodified RNA (A-RNA) and $\mathrm{m}^{6} \mathrm{~A}-\mathrm{RNA}$. Interestingly, it appears that $\mathrm{YTH}^{\mathrm{YTHDF}}$ binds to A-RNA in a one-step binding mode (with the goodness-of-fit $\mathrm{R}^{2}$ value of 0.9943 ), but to $\mathrm{m}^{6} \mathrm{~A}-\mathrm{RNA}$ in a two-step binding mode (with $\mathrm{R}^{2}$ of 0.9987 , while the $\mathrm{R}^{2}$ value is 0.9843 in one-step binding mode.) (Figure 1B, Supplementary information, Figure S1A and Table S1A). Both FP and EMSA experiments show that $\mathrm{YTH}^{\mathrm{YTHDF} 2}$ bound to $\mathrm{m}^{6} \mathrm{~A}$-RNA with much higher binding affinity than to A-RNA (Figure 1B and 1C). Note that $30 \mathrm{pmol}$ of $\mathrm{YTH}^{\mathrm{YTHDF}}$ led to an almost complete shift of $\mathrm{m}^{6} \mathrm{~A}-\mathrm{RNA}$

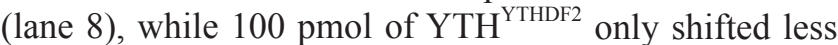
than half of the A-RNA (lane 5) (Figure 1C). A similar two-step binding mode was observed in other RNA-binding proteins [13]. It is likely that binding to RNA facilitates the further recognition of $\mathrm{m}^{6} \mathrm{~A}$.

To reveal the molecular mechanism for specific recognition of $\mathrm{m}^{6} \mathrm{~A}-\mathrm{RNA}$ by $\mathrm{YTH}^{\mathrm{YTHDF} 2}$, we solved the crystal structure of $\mathrm{YTH}^{\mathrm{YTHDF} 2}$ at $2.1 \AA$ resolution (Figure 1D). The YTH domain forms a dimer in the crystal due to crystal packing (Supplementary information, Figure S1B and Table S1B). The overall structure shows a globular fold with a central core of four-stranded $\beta$-sheets surrounded by four $\alpha$ helices and flanking regions on two sides. A Dali search indicates that $\mathrm{YTH}^{\mathrm{YTHDF} 2}$ is structurally similar to YTHDC1 (PDB: 2YUD) with a rootmean-squared deviation (rmsd) of $1.54 \AA$ for $121 \mathrm{C} \alpha$ atoms, suggesting a conserved mechanism for $\mathrm{m}^{6} \mathrm{~A}-\mathrm{RNA}$ recognition (Supplementary information, Figure S1C).

Electrostatic potential surface of $\mathrm{YTH}^{\mathrm{YTHDF} 2}$ shows a patch that is enriched in basic residues, which may be involved in RNA recognition (Figure 1E). The basic patch is formed by residues R411 and K416 on strand $\beta 1, R 441$ on helix $\alpha 2$, and R527 on the loop connecting helices $\alpha 3$ and $\alpha 4$ (Figure 1A and 1D). Close to this basic patch, a hydrophobic pocket is formed by aromatic residues Y418, W432, W486 and W491 and is supported by the loop connecting strand $\beta 1$ and helix $\alpha 1$, the loop connecting strands $\beta 3$ and $\beta 4$, and the loop connecting helices $\alpha 1$ and $\alpha 2$ (Figure 1A and 1D).

Based on above structural analyses, we further characterized several residues that are potentially important for $\mathrm{m}^{6} \mathrm{~A}-\mathrm{RNA}$ recognition. Wild-type and mutant $\mathrm{YTH}^{\mathrm{YTHDF} 2}$ were purified and used for the FP assays (Figure 1F-1G 
and Supplementary information, Table S1A). Consistent with the structural observations, K416A and R527A mutations of $\mathrm{YTH}^{\mathrm{YTHDF} 2}$ significantly decreased the binding affinity to both A-RNA ( $\sim 5$ and $\sim 10$ folds, respectively) and $\mathrm{m}^{6} \mathrm{~A}-\mathrm{RNA}$ ( $\sim 25$ folds), suggesting that these two residues are involved in binding to the backbone of RNA, but may not recognize the methyl-group of $\mathrm{m}^{6} \mathrm{~A}$. Similar results were obtained for the K416A mutant of YT$\mathrm{H}^{\mathrm{YTHDF} 2}$ in EMSA (Supplementary information, Figure S1D). In contrast, R411A and R441A mutations of YT$\mathrm{H}^{\mathrm{YTHDF} 2}$ slightly decreased RNA-binding affinity toward A-RNA ( $\sim 2$ folds) and $m^{6} \mathrm{~A}-\mathrm{RNA}$ ( $\sim 3$ folds). It is worthy to note that we calculated binding affinities of wildtype and two mutants (R411A and R441A) of $\mathrm{YTH}^{\mathrm{YTHDF} 2}$
A

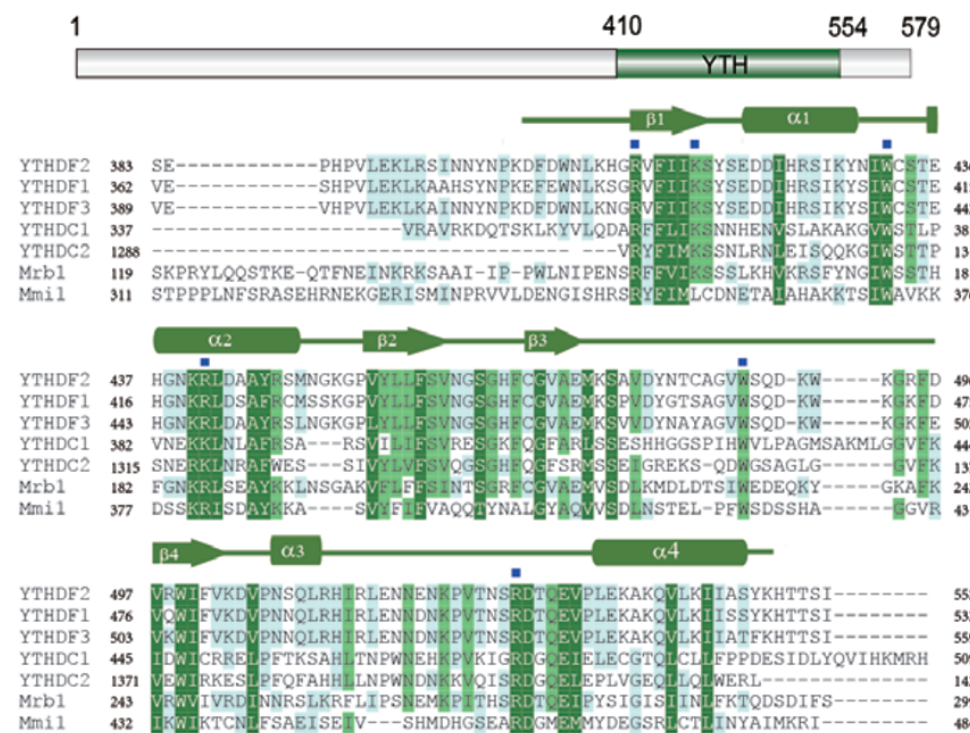

D

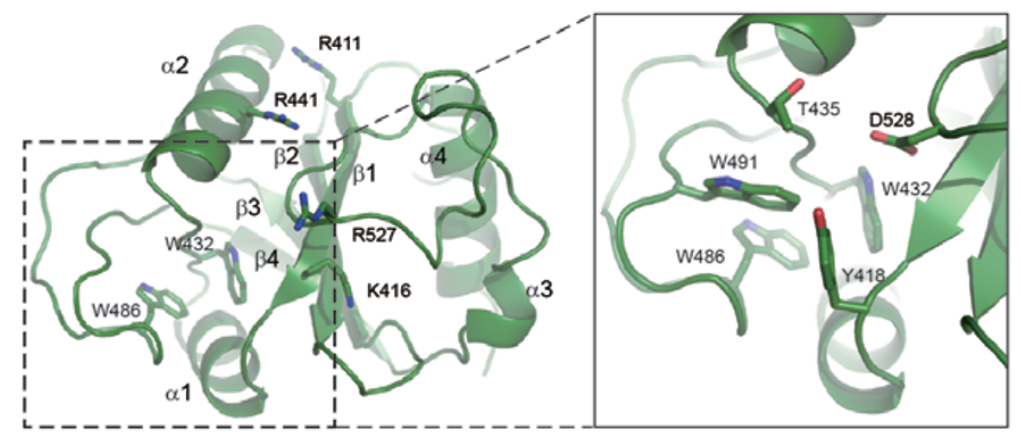

F

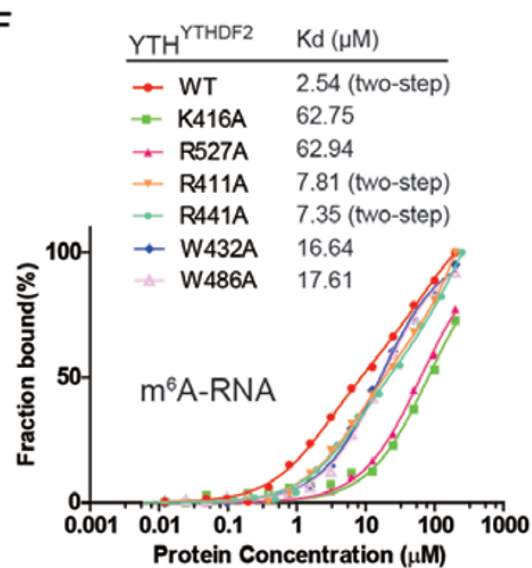

G

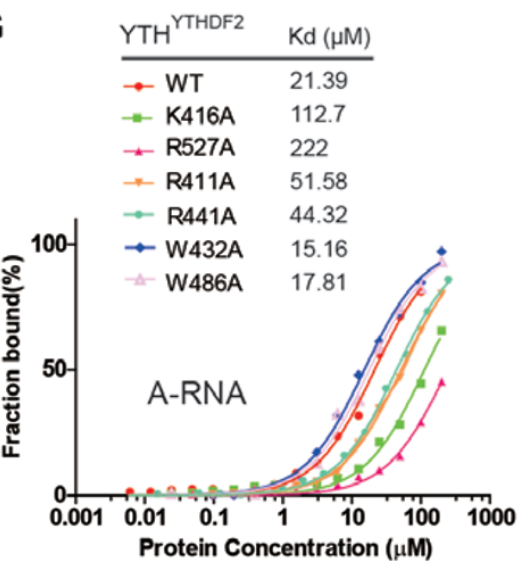

B

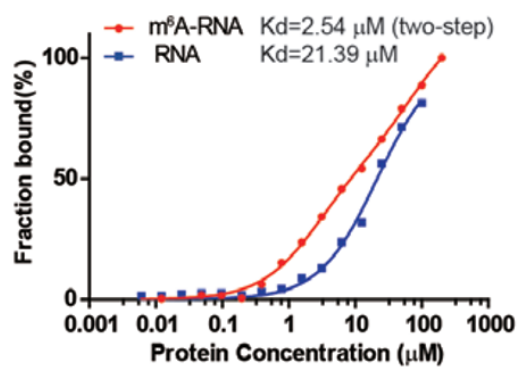

C

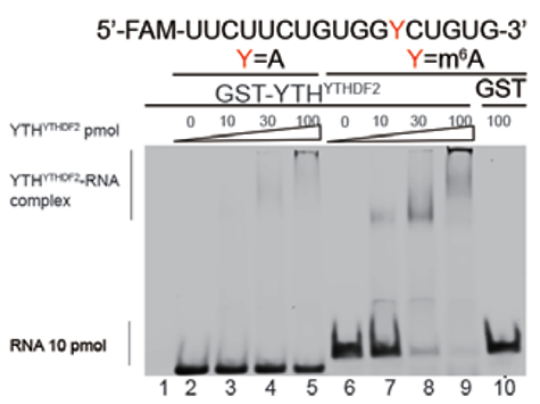

E

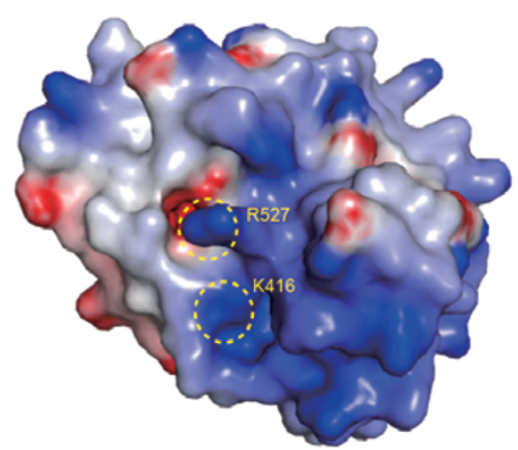

$\mathrm{H}$

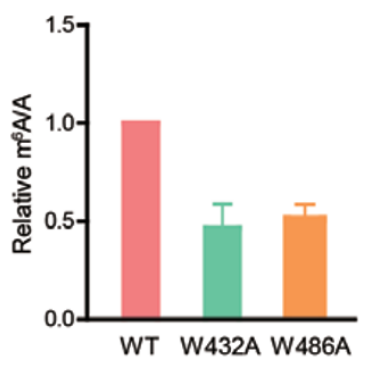


Figure 1 Crystal structure of YTHDF2 reveals mechanism for recognition of N6-methyladenosine. (A) Schematic representation of YTHDF2 (NP 057342.2) and structure-based sequence alignment of selected YTH domains. Identical and highly conserved residues are highlighted in dark green, with conserved residues in light green and less conserved ones in light blue. Secondary structural elements are indicated above the sequence. Residues that may be involved in recognition of $\mathrm{m}^{6} \mathrm{~A}-\mathrm{RNA}$ are indicated as blue squares. (B) FP assay of YTH ${ }^{\text {YTHDF2 }}$ binding to A-RNA or $\mathrm{m}^{6} \mathrm{~A}$-RNA. In the two-step binding mode, the binding affinity $(K d)$ of the first step is indicated. The $K d$ shown might be lower than the real value as binding saturation could not be achieved during the FP assays. (C) EMSA using A-RNA or $\mathrm{m}^{6} \mathrm{~A}$-RNA with increasing amount of $\mathrm{YTH}^{\mathrm{YTHDF} 2}$ proteins as indicated. (D) Ribbon representation of the crystal structure of $\mathrm{YTH}^{\mathrm{YTHDF} 2}$. A close-up view of the residues for hydrophobic pocket formation is shown on the right. Critical residues that may be involved in $\mathrm{m}^{6} \mathrm{~A}$ recognition are indicated as stick representations. (E) Electrostatic potential surface of $\mathrm{YTH}^{\mathrm{YTHDF}}$. Positive charge is colored in blue with neutral charge in white and negative charge in red. Critical residues for RNA interaction are indicated. (F-G) Superimposed FP plots of RNA-binding affinities for wild-type and mutant YTH ${ }^{\text {YTHDF2 }}$ toward $m^{6} A-R N A(F)$ or A-RNA (G). The experiments were performed as in B. (H) Relative enrichment of $m^{6} A-R N A$ in RNA products immunoprecipitated by wild-type and mutant YTHDF2 full-length proteins from HEK293T cells. The levels of RNA products were determined by LC-MS/MS. Error bars represent SD for triplicate experiments.

to $\mathrm{m}^{6} \mathrm{~A}-\mathrm{RNA}$ based on both the two-step and one-step binding modes (Supplementary information, Figure S1A and Table S1). The binding affinities of other mutants to $\mathrm{m}^{6} \mathrm{~A}-\mathrm{RNA}$ were calculated based on the one-step binding mode (Supplementary information, Table S1A).

In the FP assays, mutating two hydrophobic residues W432 and W486 to alanine markedly decreased the binding affinity of $\mathrm{YTH}^{\mathrm{YTHDF} 2}$ to $\mathrm{m}^{6} \mathrm{~A}-\mathrm{RNA}$, but barely changed the binding affinity to A-RNA, suggesting that W432 and W486 are important for specific recognition of $\mathrm{m}^{6} \mathrm{~A}$. To test the involvement of these two residues in $\mathrm{m}^{6} \mathrm{~A}$ recognition in vivo, we measured the ratio of $\mathrm{m}^{6} \mathrm{~A} /$ A levels in the RNA products immunoprecipitated by wild-type and mutant YTHDF2 full-length proteins from HEK293T cells. Indeed, W432A and W486A mutations decreased the $\mathrm{m}^{6} \mathrm{~A}-\mathrm{RNA}$ selectivity of YTHDF2. Furthermore, the W432A mutant of $\mathrm{YTH}^{\mathrm{YTHDF} 2}$ was unable to effectively pull down previously identified YTHDF2 targets, SON and CREBBP, in HeLa cells. Taken together, these data suggest that residues W432 and W486 are essential for specific recognition of $\mathrm{m}^{6} \mathrm{~A}$ by YTHDF2 (Figure $1 \mathrm{H}$ and Supplementary information, Figure S1E and S1F).

Circular dichroism (CD) measurements show that wild-type and mutant $\mathrm{YTH}^{\mathrm{YTHDF} 2}$ have similar secondary structure composition, suggesting that the overall structure of $\mathrm{YTH}^{\mathrm{YTHDF} 2}$ is not disrupted by mutations of these residues (Supplementary information, Figure S1G). Notably, residues W432 and W486 are highly conserved among the YTHDF family members from yeast to human (Figure 1A), further supporting their important role in mediating specific recognition of $\mathrm{m}^{6} \mathrm{~A}-\mathrm{RNA}$. When our manuscript was under revision, two crystal structures of $\mathrm{m}^{6} \mathrm{~A}-\mathrm{RNA}$ in complex with YTH domains from YTHDC1 [14] and Zygosaccharomyces rouxii MRB1 (ZrMRB1) [15] were reported. Structural comparison shows that residues W432 and W486 of YTH ${ }^{\mathrm{YTHDF} 2}$ adopt a similar conformation to that of $\mathrm{m}^{6} \mathrm{~A}$-binding residues in the two complex structures (Supplementary information, Figure $\mathrm{S} 1 \mathrm{H}$ ), suggesting a conserved mechanism for $\mathrm{m}^{6} \mathrm{~A}-\mathrm{RNA}$ recognition by YTH domains.

Taken together, our studies indicate that the basic residues $\mathrm{K} 416$ and $\mathrm{R} 527$ on the surface of $\mathrm{YTH}^{\mathrm{YTHDF} 2}$ are involved in binding to the RNA backbone, and residues W432 and W486 within the hydrophobic pocket contribute to the specific recognition of $\mathrm{m}^{6} \mathrm{~A}$. Our study also provides a platform for further investigations of additional YTH-m ${ }^{6} \mathrm{~A}-\mathrm{RNA}$ complex structures, which would reveal molecular mechanisms for specific recognition of $\mathrm{m}^{6} \mathrm{~A}-\mathrm{RNA}$ by YTHDF2 and other YTH family proteins.

The coordinate and structure factor for the $\mathrm{YTH}^{\mathrm{YTHDF} 2}$ structure have been deposited into the Protein Data Bank under the accession code of $4 \mathrm{WQN}$.

\section{Acknowledgments}

We thank staff members of beamline BL17U at SSRF (Shanghai Synchrotron Radiation Facility, China) for their assistance in data collection, and Mr Lei Zhang and staff members of Biomedical Core Facility in Fudan University for their help on biochemical analyses. We thank Dr Jinbiao Ma for the help on synthesis of $\mathrm{m}^{6} \mathrm{~A}-\mathrm{RNA}$. This work was supported by grants from the National Basic Research Program of China (2011CB965300), the National Science \& Technology Major Project "Key New Drug Creation and Manufacturing Program" of China (2014ZX09507-002), the National Natural Science Foundation of China (U1432242, 91419301, 31270779 and 31030019), the Basic Research Project of Shanghai Science and Technology Commission (12JC1402700), and the "Shu Guang" project (11SG06) supported by Shanghai Municipal Education Commission and Shanghai Education Development Foundation.

Tingting $\mathrm{Zhu}^{1}$, Ian A Roundtree ${ }^{2}$, Ping Wang ${ }^{1}$, Xiao Wang ${ }^{3,4}$, Li Wang ${ }^{1}$, Chang Sun ${ }^{1}$, Yuan Tian ${ }^{1}$, Jie $\mathrm{Li}^{1}$, Chuan $\mathrm{He}^{3,4}$, Yanhui $\mathrm{Xu}^{1}$ 
${ }^{I}$ Fudan University Shanghai Cancer Center, Department of Oncology; and Institute of Biomedical Sciences and School of Basic Medical Sciences, Shanghai Medical College of Fudan University, Shanghai 200032, China; ${ }^{2}$ Medical Scientist Training Program and Department of Biochemistry and Molecular Biology, The University of Chicago, Chicago, IL 60637, USA; ${ }^{3}$ Department of Chemistry and Institute for Biophysical Dynamics, The University of Chicago, Chicago, IL 60637, USA; ${ }^{4}$ Howard Hughes Medical Institute, The University of Chicago, Chicago, IL 60637, USA

Correspondence: Yanhui $\mathrm{Xu}$

E-mail: xuyh@fudan.edu.cn

\section{References}

1 Fu Y, Dominissini D, Rechavi G, He C. Nat Rev Genet 2014; 15:293306.

2 Schwartz S, Mumbach MR, Jovanovic M, et al. Cell Rep 2014; 8:284296.
3 Ping XL, Sun BF, Wang L, et al. Cell Res 2014; 24:177-189.

4 Jia G, Fu Y, Zhao X, et al. Nat Chem Biol 2011; 7:885-887.

5 Liu J, Yue Y, Han D, et al. Nat Chem Biol 2014; 10:93-95.

6 Zheng G, Dahl JA, Niu Y, et al. Mol Cell 2013; 49:18-29.

7 Fustin JM, Doi M, Yamaguchi Y, et al. Cell 2013; 155:793-806.

8 Dominissini D, Moshitch-Moshkovitz S, Schwartz S, et al. Nature 2012; 485:201-206.

9 Schwartz S, Agarwala SD, Mumbach MR, et al. Cell 2013; 155:14091421.

10 Wang Y, Li Y, Toth JI, et al. Nat Cell Biol 2014; 16:191-198.

11 Wang X, Lu Z, Gomez A, et al. Nature 2014; 505:117-120.

12 Nguyen TT, Ma LN, Slovak ML, et al. Genes Chromosomes Cancer 2006; 45:918-932.

13 Zucconi BE, Ballin JD, Brewer BY, et al. J Biol Chem 2010; 285:39127-39139.

14 Xu C, Wang X, Liu K, et al. Nat Chem Biol 2014; 10:927-929.

15 Luo S, Tong L. Proc Natl Acad Sci USA 2014; 111:13834-13839.

(Supplementary information is linked to the online version of the paper on the Cell Research website.) 